\title{
Changes across the psychometric function following perceptual learning of an RSVP reading task
}

\author{
Daniel R. Coates ${ }^{1 *}$ and Susana T. L. Chung ${ }^{1,2}$ \\ Vision Science Graduate Program, University of California, Berkeley, CA, USA \\ 2 School of Optometry, University of California, Berkeley, CA, USA
}

\author{
Edited by: \\ Gianluca Campana, Università degli \\ Studi di Padova, Italy \\ Reviewed by: \\ David P. McGovern, Trinity College \\ Institute of Neuroscience, Ireland \\ Kenneth Knoblauch, Institut National \\ de la Santé et Recherche Médicale, \\ France \\ *Correspondence: \\ Daniel R. Coates, Vision Science \\ Graduate Program, University of \\ California, Minor Hall, Berkeley, CA \\ 94720-2020, USA \\ e-mail:daniel.coates@berkeley.edu
}

\begin{abstract}
Several recent studies have shown that perceptual learning can result in improvements in reading speed for people with macular disease (e.g., Chung, 2011; Tarita-Nistor et al., 2014). The improvements were reported as an increase in reading speed defined by specific criteria; however, little is known about how other properties of the reading performance or the participants' perceptual responses change as a consequence of learning. In this paper, we performed detailed analyses of data following perceptual learning using an RSVP (rapid serial visual presentation) reading task, looking beyond the change in reading speed defined by the threshold at a given accuracy on a psychometric function relating response accuracy with word exposure duration. Specifically, we explored the statistical characteristics of the response data to address two specific questions: was there a change in the slope of the psychometric function and did the improvements in performance occur consistently across different word exposure durations? Our results show that there is a general steepening of the slope of the psychometric function, leading to non-uniform improvements across stimulus levels.
\end{abstract}

Keywords: perceptual learning, reading, rapid serial visual presentation, central vision loss, psychometric functions

\section{INTRODUCTION}

Performance for a variety of visual tasks improves with practice. This improvement is often termed perceptual learning and can be observed in the normal fovea (e.g., McKee and Westheimer, 1978; Ball and Sekuler, 1982, 1987; Karni and Sagi, 1991; Poggio et al., 1992; Fahle and Edelman, 1993; Li et al., 2004; Lu and Dosher, 2004) and the periphery (e.g., Chung et al., 2004, 2005; Chung, 2007). The effectiveness of perceptual learning in improving visual performance in the periphery is particularly important in relation to visual rehabilitation because it is commonly believed that the properties of vision in people with strabismic amblyopia resemble those of the normal periphery (Levi, 1991; Levi and Carkeet, 1993), and that people who lose their central vision due to macular disease must use their peripheral vision for seeing. Indeed, perceptual learning has been used as a remedy to improve functional vision for people with amblyopia for over two decades (for reviews, see Levi, 2005, Levi and Li, 2009 or Astle et al., 2011). Only recently have we observed an intense interest in applying perceptual learning to improve functional vision in people with central vision loss. Enhancing reading performance is a central goal, likely because the majority of patients seeking low vision rehabilitation services had central vision loss and most of them complained of reading difficulties (Owsley et al., 2009).

Previously, we tested the feasibility of using perceptual learning to improve reading speed for a group of six participants with long-standing macular disease: age-related macular degeneration (AMD) or Stargardt disease (Chung, 2011). Our task involved presenting sentences one word at a time using the rapid serial visual presentation (RSVP) paradigm and measuring reading accuracy as a function of word exposure duration. The advantage of using RSVP to train people with macular disease is that RSVP minimizes the need to make intra-word saccades during reading (Rubin and Turano, 1994), thus the training is not contaminated by any potential deficiency in eye movements, as has been reported for these individuals (White and Bedell, 1990). As such, the RSVP paradigm also allows us to independently test whether eye movement training or reading training would be more beneficial to people with macular disease, as in the study of Seiple et al. (2011). For example, it is hypothesized that "crowding" between letters in the periphery limits reading speed, and it has been shown previously that peripheral letter crowding can be reduced with perceptual learning (Chung et al., 2004; Chung, 2007).

Using our method, we defined reading speed based on the word exposure duration that yielded $80 \%$ correct on the psychometric function $(\mathrm{PF})$ relating reading accuracy with word exposure duration. Our result showed that reading speed improved by an average of 53\% following 6 weekly sessions of training using an RSVP reading task. Nguyen et al. (2011) trained a group of Stargardt disease patients also using an RSVP reading task and reported an increase of $25 \%$ of the median reading speed of the group following training. More recently, Tarita-Nistor et al. (2014) reported a 54\% improvement in reading speed following training with a print size close to the threshold print size. In all these studies, reading speed was the primary measurement during training, and was used to gauge the effectiveness of the training paradigm. Besides the improvement in reading speed, which often was defined based on the shortest amount of time to read 
at a given level of accuracy, little is known about whether or not, and how, perceptual learning alters other properties of the participants' reading responses. Did the improvement in reading speed occur only at a specific testing duration, or did it generalize to other durations?

To further probe the effects of perceptual learning on the properties of participants' reading responses, we need to be able to fully characterize participants' reading performance at different stimulus levels (reading durations) and/or accuracy levels. The approach in our previous paper, measuring reading performance using the method of constant stimuli for multiple word durations, and fitting a psychometric function of reading accuracy vs. stimulus duration to describe the data (Chung, 2011), offers us the opportunity to explore the statistical characteristics of the response data beyond the defined reading speed thresholds. Other approaches have also been used to measure RSVP reading performance, most commonly using adaptive methods (Nguyen et al., 2011; Seiple et al., 2011; Tarita-Nistor et al., 2014). These approaches target at trials around a given accuracy criterion (the "threshold") and do not lend themselves readily for analyses beyond giving us the threshold values. In this paper, we are specifically interested in two questions: (1) was there a change in the slope of the psychometric function as a result of perceptual learning, and (2) were the performance improvements uniform across different word exposure durations?

To address our questions, we performed detailed analyses on the dataset of Chung (2011), with data from an additional new participant, also with age-related macular degeneration (AMD). Because a psychometric function was used to fit the data from each training block, we could evaluate if the slope of the psychometric functions change over the learning process (Question 1). An understanding of changes in the slope of the psychometric function is critical for at least two reasons. First, assumptions about the slope are the theoretical basis of adaptive methods such as QUEST (Watson and Pelli, 1983; Kontsevich and Tyler,
1999). Second, changes in the slope of the underlying psychometric function with learning may provide information about the underlying mechanism of the learning process or the specificity of the learning effect.

The predictions of how a psychometric function relating reading accuracy and stimulus duration may change as a result of perceptual learning are shown in Figure 1. The blue and red curves in each panel represent the psychometric function before and after training, respectively. Here, we make two general assumptions of the effects of perceptual learning on reading performance: (1) reading speed improves, which means that at the same level of accuracy, words can be read at shorter durations after training than before; and (2) the slope of the psychometric function either remains the same or becomes steeper after training, but will not become shallower. The three scenarios in Figure 1 summarize how these effects may combine to produce the observed changes in the psychometric function following training. Panel A shows the scenario in which the slope of the psychometric function (sensitivity of responses) does not change. In this case, improvements in reading performance appear as a mere leftward shift of the psychometric function toward shorter durations (corresponding to faster reading speeds), yielding similar magnitudes of improvements across all durations, except at the very low and high end of the psychometric function. Panel B represents the case in which only the slope of the psychometric function becomes steeper (improvement in response sensitivity) following training. Because the slope of a psychometric function is defined with respect to the mean of the function (the $50 \%$ point), a steepening of the psychometric function (without any horizontal shift) would appear as an improvement in reading speed for accuracy levels above the mean of the psychometric function. However, this scenario predicts that there will be a drop in reading speed corresponding to accuracy levels below the $50 \%$ point. In Panel C, the steeper psychometric function is also accompanied by a leftward shift, resulting in improvements in reading speed that differ depending

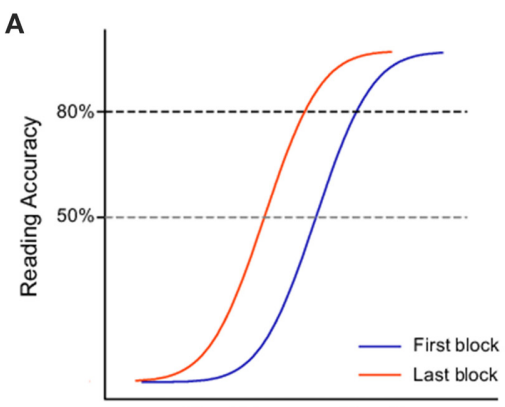

Word Exposure Duration

No change in slope of PF

Reading speeds improve by the same amount at $80 \%$ and $50 \%$ correct criterion

Reading speed improves similarly across most durations
B

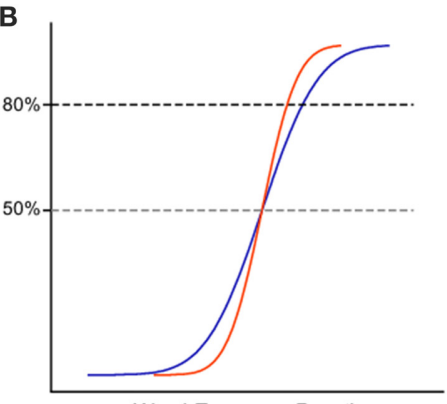

Word Exposure Duration

Slope of PF becomes steeper

Reading speed improves at $80 \%$ correct criterion, but no change at $50 \%$-correct criterion

Reading speed improves at \%correct criteria $>50 \%$ but drops at criteria $<50 \%$-correct

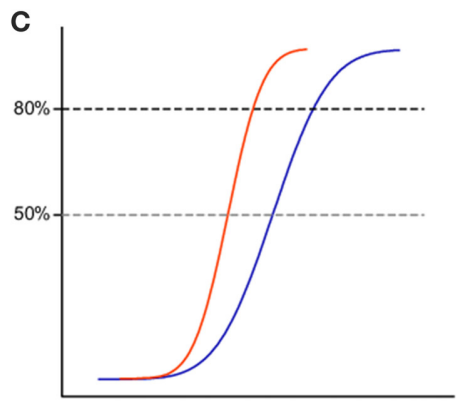

Word Exposure Duration

Slope of PF becomes steeper Reading speed improves more at $80 \%$ than at $50 \%$-correct criterion Reading speed improves more at high \%-correct criteria than at lowcorrect criteria

FIGURE 1 | Panels A-C illustrate the three possible outcomes of perceptual learning. 
on the accuracy levels. For instance, reading speed defined at an $80 \%$ correct accuracy would yield a larger improvement than at $50 \%$ correct.

\section{MATERIALS AND METHODS \\ 2.1. EXPERIMENTAL PROCEDURES}

Details of the experimental procedures are provided in Chung (2011). In brief, seven participants with macular disease practiced reading for six training sessions. Participants S1-S6 were from the study of Chung (2011), while data from S7 has not been reported previously. Visual characteristics of these seven participants are given in Table 1. Before and after training, participants were tested on a battery of tests that included the measurements of visual acuity, the location of the preferred retinal locus for fixation, fixation stability, the critical print size for reading and the maximum reading speed (when print size is not a limiting factor). The post-pre changes of these tests, if any, were reported previously in Chung (2011). In this paper, we focus on reporting the changes of the psychometric functions as a result of perceptual learning.

Reading performance was assessed using oral reading speed for single sentences presented in the RSVP format (Chung et al., 1998; Chung, 2002, 2011). On each trial, a single sentence was chosen randomly from a pool of 2630 sentences, containing between 8 and 14 words (mean $=10.9 \pm 1.7$ [SD]). All the words used were among the 5000 most frequently used words in written English, according to word-frequency tables derived from the British National Corpus (Kilgarriff, 1997). Words were rendered in Times-Roman font and were presented left-justified on a computer display, one word at a time in rapid succession, each for a fixed exposure duration. Participants were asked to read the words as quickly and as accurately as possible. The number of words read correctly was recorded after each trial. Feedback as to the number of words read correctly or the correct words (if read incorrectly) was not provided. In each block of trials, we used the method of constant stimuli to present sentences at five word exposure durations. The durations were chosen such that participants' reading accuracy spanned a range from $0-10 \%$ to $90-100 \%$ correct. Six sentences were tested at each duration, with a total of 30 sentences tested in each block and in a random order. With the exception of S6, all participants completed 10 blocks of trials (30 trials, or an average of $\sim 330$ words presented per block) in each

Table 1 | Participant demographics.

\begin{tabular}{lcclrlc}
\hline Participant & Sex & Age & Diagnosis & $\begin{array}{c}\text { LogMAR } \\
\text { Acuity (RE, LE) }\end{array}$ & $\begin{array}{c}\text { In 2011 } \\
\text { study? }\end{array}$ \\
\hline S1 & F & 82 & AMD & 0.50 & 0.52 & Yes \\
S2 & M & 85 & AMD & 0.70 & 0.74 & Yes \\
S3 & M & 84 & AMD & 0.56 & 0.70 & Yes \\
S4 & F & 73 & AMD & 0.66 & 0.48 & Yes \\
S5 & F & 62 & Stargardt & 0.58 & 0.58 & Yes \\
S6 & M & 57 & Stargardt & 1.10 & 1.10 & Yes \\
S7 & M & 72 & AMD & 0.78 & 0.78 & No \\
\hline
\end{tabular}

All characteristics listed refer to the values during participation in the study. of the six training sessions, for a total of 60 blocks. S6 completed only seven blocks in the first training session, and eight in each of the subsequent sessions, for a total of 47 blocks. Training sessions were scheduled once a week for six consecutive weeks for participants S1-S5. Due to unexpected illness and personal issue, there was a three-week gap between sessions 3 and 4 for S6, the rest of his training sessions also occurred on a weekly basis. S7's training occurred on a daily basis (due to availability of the participant). Previously we have reported that the improvements due to perceptual learning are similar whether training took place on a daily or a weekly basis (Chung and Truong, 2013). With the exception of the frequency of training sessions (daily vs. weekly), the training protocol was identical for all participants.

\section{RESULTS}

\subsection{STATISTICAL MODELING}

To perform the statistical analyses subsequently described, we used the free software R ( $\mathrm{R}$ Core Team, 2014). Additional analysis and plotting routines were written in Python, using the IPython (Pérez and Granger, 2007) environment and the NumPy/SciPy mathematics libraries (Millman and Aivazis, 2011).

There are several ways to analyze how the parameters of the psychometric function change over the course of training. The traditional approach (widely used, including in our own previous study), is to fit a psychometric function in each block as the first step, and then compare (i.e., with ANOVA, $t$-tests, etc.) or otherwise process the results of the fits (such as smoothing, fit an exponential, etc.) This two-step procedure is called the Parameter-As-Outcome Model (PAOM) in a recent article (Moscatelli et al., 2012) which serves as a tutorial to an alternative method comprising a principled one-step approach.

Using the one-step technique, psychometric functions are simultaneously fit to data and processed over time in a single step, permitting more robust statistical analyses. Since the change in performance over time is best described by an exponential function (Dosher and Lu, 2007; Chung, 2011), a non-linear mapping of the parameters vs. training block must be employed. No hypotheses exist about the change in slope over training, so non-parametric, assumption-free methods must be used. There are several possibilities, such as "additive models" (Wood, 2006), which have advantages over other non-parametric approaches such as LOESS or kernel regression (Knoblauch and Maloney, 2012). In a similar vein, we employed orthogonal polynomial fitting, where the change over time is modeled using sums of polynomials of increasing powers of the predictor variable (block number). Using $\mathrm{R}$, this method can be incorporated as described above with simultaneous fitting of the psychometric function at each block, with the typical assumptions of a cumulative Gaussian psychometric function and binomial variance, such as in traditional probit analysis.

Two variants of this model were tested. The more general model (denoted $\mathrm{M}_{v a r}$ ) models both the slope and 50\% point as arbitrary functions of the block number. An alternative model $\left(\mathrm{M}_{\text {fixed }}\right)$, lets only the $50 \%$ point vary with the block; the slope is fixed. The sole free parameter when using orthogonal polynomials is the highest order of polynomial to utilize in fitting. Higher orders always yield a better fit to the data, but the risk is 
overfitting noise. To account for this, when choosing an order, a statistic such as BIC (Bayesian Information Criteria) is computed that penalizes the model likelihood by the number of parameters (Knoblauch and Maloney, 2012), here the highest order of polynomial. We summed the BIC across participants and evaluated all possible orders (1-60) of model $\mathrm{M}_{\text {var }}$. The minimum BIC occurred at order 2 , meaning the slope and $50 \%$ point are best approximated by the sum of a linear term and a quadratic term. A third model $\left(\mathrm{M}_{\text {exp }}\right)$ extended the traditional analysis (such as Dosher and Lu, 2007; Chung, 2011), modeling the change in performance as an exponential that reaches an asymptote, combined with a potential change in slope modeled as an arbitrary function, again using orthogonal polynomials.

\subsection{PARAMETER ESTIMATES: CHANGES IN SLOPE AND 50\% POINT}

Figures 2, 3 depict the estimated values for the 50\% point and slope, respectively. Each line shows the fit based on the method indicated in the figure legend. Several qualitative observations can be made about Figure 2. First, despite the difference in the constraints of each of the three models, the $50 \%$ point estimates are remarkably consistent between models. Most participants show a decrease in stimulus duration corresponding to the $50 \%$ point over the duration of training. The decrease is generally asymptotic, resulting in a function that is concave upward. S1 shows little improvement, however, and S4 may not have reached asymptotic performance. Figure 3 demonstrates a general increase (steepening) in the slope of the psychometric
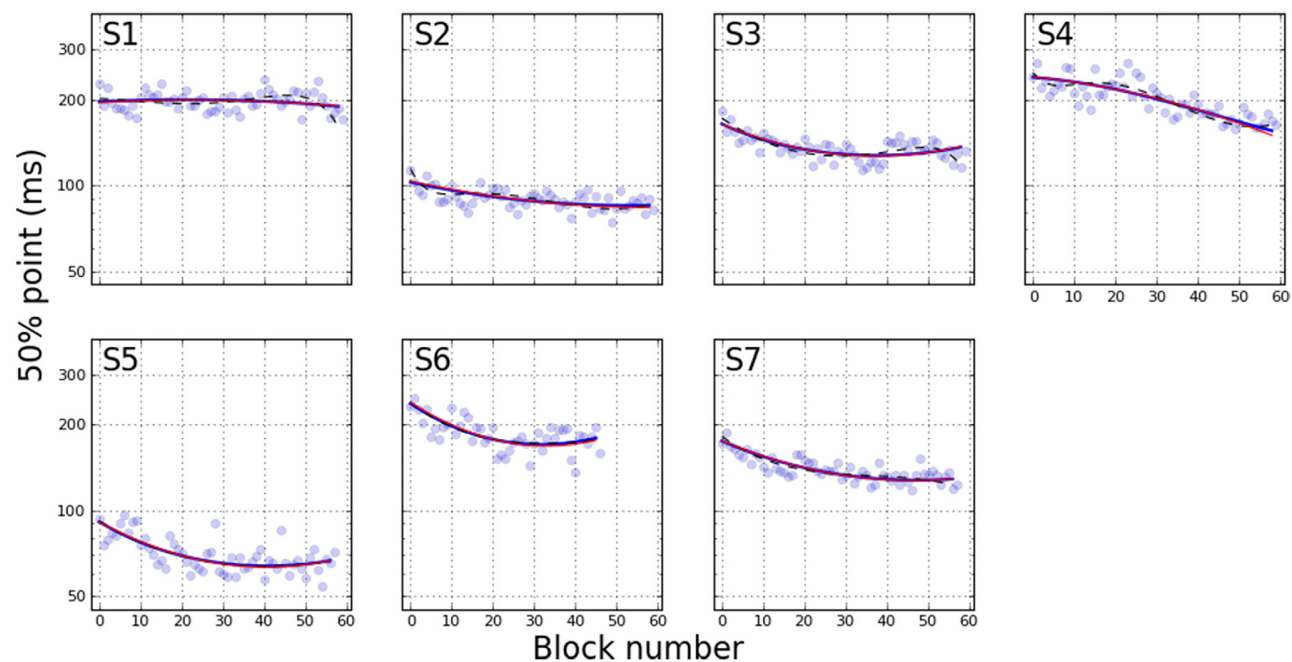

FIGURE 2 | $50 \%$ point on psychometric function as a function of training block. Blue curves are estimated using the variable slope model, while red curves indicate fixed slope. These curves are

nearly indistinguishable. Dashed black lines show exponential fit. Pale blue dots represent PFs fit independently to each individual block.
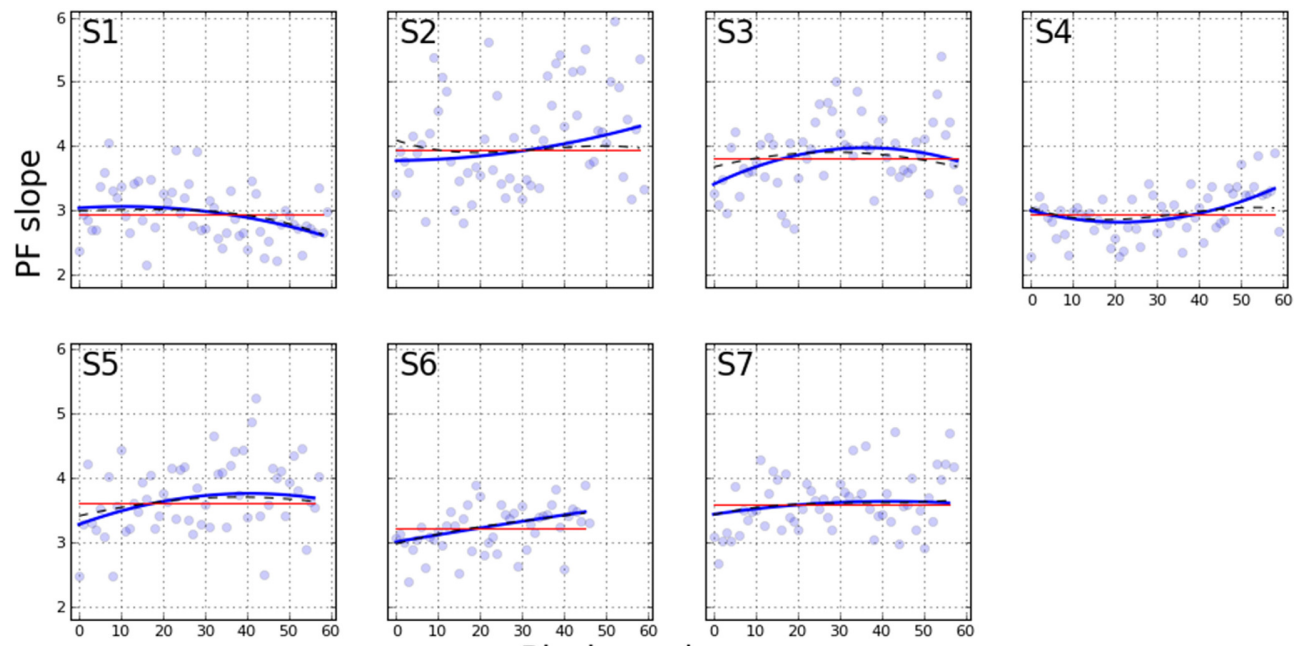

\section{Block number}

FIGURE 3 | Slope of PF across training blocks. Curves are colored as in Figure 2, with the estimated slope parameter as the variable of interest. 
functions, either asymptotic (S3, S5, S7), or increasing (S2, S4, S6). S1 is the only participant showing a decrease (shallowing) of the slope. These trends are generally consistent between the two models that let the slope vary, while the slope for model $\mathrm{M}_{\text {fixed }}$ is flat, by definition.

To compare with Chung (2011), Figure 4 plots the "reading speed" that is calculated from the estimated PFs. Reading speed is defined as the duration yielding $80 \%$ correct, converted to wordsper-minute $(\mathrm{wpm})$ by dividing the duration into $60 \times 1000$. This graph can be compared directly to Figure 1 of Chung (2011). For the present study, the only real divergence between the three models can be observed with S4. Here the orthogonal polynomials estimate the change in reading speed as an upward concave function, whereas the exponential fit models the change as a shallow linear curve. Another difference between the exponential fit and the other smoothing approaches is that $\mathrm{S} 1$ does not show an improvement in reading speed except for a steep rise in the first few blocks, which is best captured by the exponential.

The improvements due to perceptual learning can be quantified by comparing the fitted values from the first and last blocks, as shown in Tables 2-4. These tables demonstrate the similarity of the predictions of the three models, as well as indicating the ratio of improvement from first to last block.
Table 4 further demonstrates the reasonable agreement in reading speed measurement with Chung (2011), despite the difference in methods.

Until now, we have reported the changes in $50 \%$ point and the slope of the psychometric functions as separate entities, but since both parameters progressively change with training, are these values related? Figure $\mathbf{5}$ shows the corresponding changes to both the $50 \%$ point and psychometric function slope for all 7 participants on a single plot, as estimated using $\mathrm{M}_{\text {var }}$. Each marker indicates the value of the two PF parameters on one training block, with color indicating participant and symbol size going from small to large to indicate the progression of training blocks. For most participants, it is clear that a steepening of the psychometric function accompanied the observed decrease in $50 \%$ point, though there are significant individual differences.

\subsection{ESTIMATED CHANGE IN PERFORMANCE ACROSS THE PF}

Changes in RSVP reading speed with training has been reported previously, but to our knowledge, whether or not the slope of the psychometric function changes with training has not been established. To confirm that the slope change is indeed significant, we performed statistical model comparison of the $\mathrm{M}_{\text {var }}$ and $\mathrm{M}_{\text {fixed }}$ models. Since $\mathrm{M}_{\text {fixed }}$ is a nested model of $\mathrm{M}_{\text {var }}$,
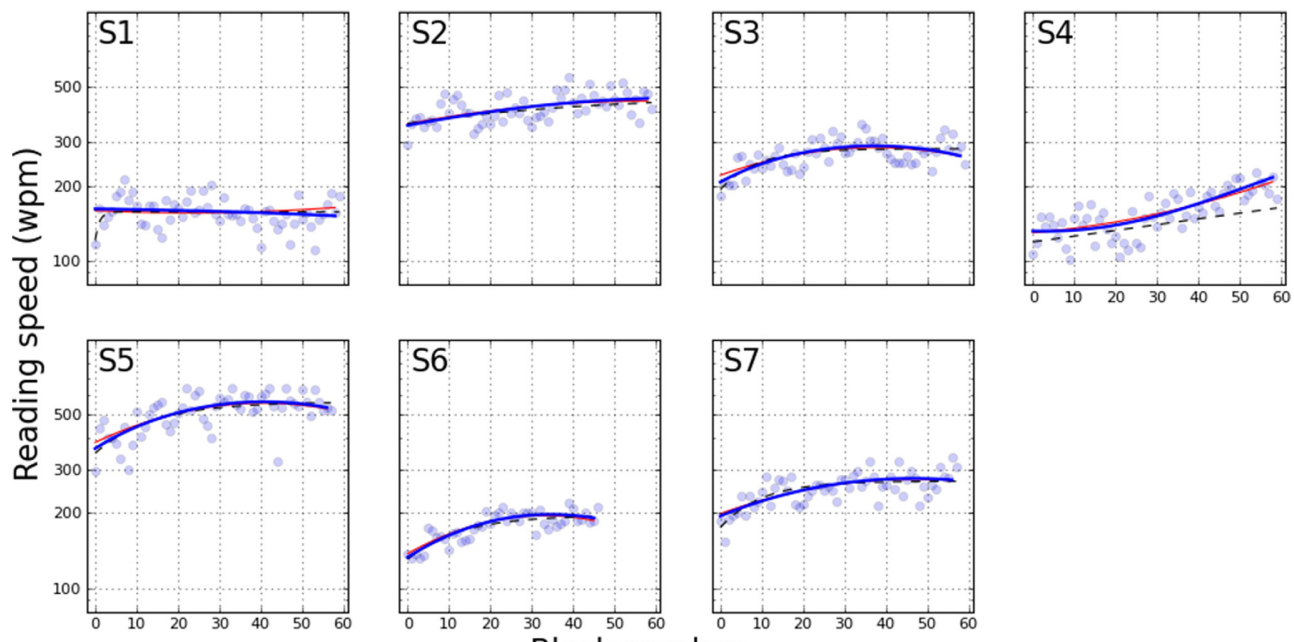

Block number

FIGURE 4 | Reading speed across training blocks. The data is derived from Figures 2, 3, with conversion specified as reading speed $=\frac{60 \times 1000}{80 \% \text { accuracylevel }}$

Table 2 | Estimated first and last PF $\mathbf{5 0 \%}$ points.

\begin{tabular}{|c|c|c|c|c|c|c|c|c|c|}
\hline Participant & \multicolumn{3}{|c|}{ First block } & \multicolumn{3}{|c|}{ Last block } & \multicolumn{3}{|c|}{ Ratio $\left(\frac{\text { First }}{\text { Last }}\right)$} \\
\hline $\mathrm{S} 2$ & 102.58 & 103.40 & 113.95 & 85.16 & 83.58 & 85.85 & 1.20 & 1.24 & 1.33 \\
\hline S3 & 164.16 & 163.61 & 171.99 & 136.07 & 136.64 & 117.09 & 1.21 & 1.20 & 1.47 \\
\hline S4 & 239.02 & 239.86 & 247.82 & 155.30 & 149.64 & 162.96 & 1.54 & 1.60 & 1.52 \\
\hline S6 & 237.02 & 239.22 & 239.57 & 179.43 & 176.35 & 176.26 & 1.32 & 1.36 & 1.36 \\
\hline S7 & 175.30 & 175.51 & 182.05 & 128.99 & 129.13 & 123.17 & 1.36 & 1.36 & 1.48 \\
\hline
\end{tabular}


a straightforward $\chi^{2}$ difference test can be used. With this analysis, the addition of the two slope terms (the linear and quadratic coefficients), was statistically significant for all participants except S7. (For this participant, note the flatness of the estimated slope in Figure 3). Table 5 lists the results of this test for each participant.

The change in slope is consistent amongst observers except for S1 (opposite sign of slope change) and S7 (not statistically significant). It is well known that there is substantial individual variability in the effects of perceptual learning (Fahle and Henke-Fahle, 1996), therefore we are not surprised that not all participants showed the same effects. In fact, the percentage of our participants not showing the effect as the other participants ( $\sim 28 \%)$ is comparable to the values reported for the percentage of participants not showing any improvement following perceptual learning (Fahle and Henke-Fahle, 1996; Chung et al., 2005).

To clearly illustrate the change in performance across the psychometric function, Figure 6 shows the first and last PF for each participant. The full PFs are shown (estimated using model $\mathrm{M}_{v a r}$ ), as well as the empirical data for the two blocks. From the PFs, the expected ratio of performance improvements can be estimated for any arbitrary criteria, as shown in Figure 7. Clearly, the more similar the slopes are, the flatter the ratio curve at different points of the psychometric function. In the case where the slope does not change (Figure 1A), the improvement curve will be completely flat (uniform improvements across $\mathrm{PF}$ ), whereas for the steepening slope case (Figure 1C), the curve shown in Figure 7 will increase for higher performance levels (larger $\times$ values). Finally, the case of Figure 1B would result in a curve with lower performance levels worsening $($ ratio $<1)$, and higher performance levels improving (ratio $>1$ ). For our participants, S2-S6 exhibited a pattern consistent with Figure 1C, in agreement with the slope ratios shown in Table 3. S1 showed a negative pattern, while S7's pattern was flat, more consistent with Figure 1A.

\section{DISCUSSION}

In this paper, we performed detailed analyses of the rich data set of Chung (2011), with additional data from another participant

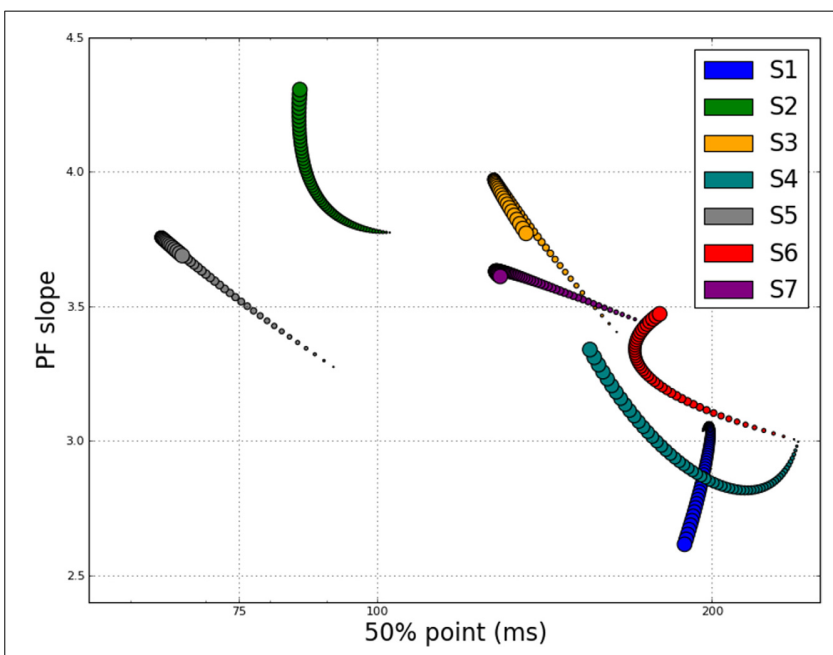

FIGURE 5 | Slope of PF vs. $\mathbf{5 0} \%$ point for each block of each participant, showing evolution throughout training. Color indicates participant, with circle size going from small to large over course of training.

Table 3 | Estimated first and last PF slopes.

\begin{tabular}{|c|c|c|c|c|c|c|c|c|c|}
\hline \multirow[t]{2}{*}{ Participant } & \multicolumn{3}{|c|}{ First block } & \multicolumn{3}{|c|}{ Last block } & \multicolumn{3}{|c|}{ Ratio $\left(\frac{\text { First }}{\text { Last }}\right)$} \\
\hline & $\mathrm{M}_{\text {var }}$ & $M_{f i x e d}$ & $M_{\exp }$ & ${ }^{M_{v a r}}$ & $M_{\text {fixed }}$ & $M_{\exp }$ & $\mathbf{M}_{\text {var }}$ & $M_{\text {fixed }}$ & $M_{\text {exp }}$ \\
\hline S1 & 3.04 & 2.93 & 3.00 & 2.62 & 2.93 & 2.63 & 0.86 & 1.00 & 0.88 \\
\hline S2 & 3.77 & 3.94 & 4.09 & 4.31 & 3.94 & 3.97 & 1.14 & 1.00 & 0.97 \\
\hline S3 & 3.40 & 3.81 & 3.67 & 3.77 & 3.81 & 3.68 & 1.11 & 1.00 & 1.00 \\
\hline S4 & 3.00 & 2.94 & 3.04 & 3.34 & 2.94 & 3.04 & 1.11 & 1.00 & 1.00 \\
\hline S5 & 3.28 & 3.61 & 3.41 & 3.69 & 3.61 & 3.63 & 1.13 & 1.00 & 1.07 \\
\hline S6 & 3.01 & 3.22 & 2.97 & 3.47 & 3.22 & 3.45 & 1.16 & 1.00 & 1.16 \\
\hline S7 & 3.43 & 3.58 & 3.43 & 3.61 & 3.58 & 3.65 & 1.05 & 1.00 & 1.07 \\
\hline
\end{tabular}

Table 4 | Estimated first and last reading speeds, calculated as estimated $80 \%$ point on PF converted to WPM using WPM $=\frac{60 \times 1000}{d u r a t i o n}$. Columns marked (2011) show results of Chung (2011) study, which used a different method of fitting.

\begin{tabular}{|c|c|c|c|c|c|c|c|c|c|c|c|c|}
\hline \multirow[t]{2}{*}{ Participant } & \multicolumn{4}{|c|}{ First block } & \multicolumn{4}{|c|}{ Last block } & \multicolumn{4}{|c|}{ Ratio ( $\left.\frac{\text { First }}{\text { Last }}\right)$} \\
\hline & ${ }^{M_{v a r}}$ & $M_{\text {fixed }}$ & ${ }^{M} \exp$ & (2011) & $\mathrm{M}_{\text {var }}$ & $\mathrm{M}_{\text {fixed }}$ & $M_{\text {exp }}$ & (2011) & ${ }^{M_{\text {var }}}$ & $M_{\text {fixed }}$ & $M_{\exp }$ & (2011) \\
\hline S1 & 161.55 & 158.96 & 155.91 & 118.52 & 151.38 & 163.46 & 178.50 & 172.81 & 0.94 & 1.03 & 1.14 & 1.46 \\
\hline $\mathrm{S} 2$ & 350.03 & 354.81 & 327.89 & 341.92 & 449.22 & 438.94 & 428.87 & 458.19 & 1.28 & 1.24 & 1.31 & 1.34 \\
\hline S3 & 206.87 & 220.43 & 205.83 & 189.08 & 263.77 & 263.94 & 302.67 & 285.91 & 1.28 & 1.20 & 1.47 & 1.51 \\
\hline S4 & 131.49 & 129.49 & 128.07 & 126.88 & 216.24 & 207.58 & 194.48 & 195.17 & 1.64 & 1.60 & 1.52 & 1.54 \\
\hline S5 & 363.64 & 385.03 & 369.99 & 349.78 & 531.45 & 525.69 & 535.64 & 595.51 & 1.46 & 1.37 & 1.45 & 1.70 \\
\hline S6 & 132.85 & 137.50 & 130.50 & 125.13 & 191.36 & 186.53 & 194.20 & 201.94 & 1.44 & 1.36 & 1.49 & 1.61 \\
\hline S7 & 194.64 & 198.87 & 187.18 & $\mathrm{n} / \mathrm{a}$ & 271.99 & 270.30 & 286.43 & $\mathrm{n} / \mathrm{a}$ & 1.40 & 1.36 & 1.53 & $\mathrm{n} / \mathrm{a}$ \\
\hline
\end{tabular}


with $A M D$, to address questions of whether there is a change in the slope of the psychometric function as a result of perceptual learning. With respect to this question, we had a priori reason to hypothesize that the slope of the psychometric function should either remain the same (but this should be accompanied by a shift of the psychometric function to reflect improvements in performance) or become steeper following perceptual learning. Here, the slope of a psychometric function represents the magnitude of the word duration that needs to be changed in order to alter the participant's reading accuracy by a certain amount. With perceptual learning, it is expected that participants would require a smaller change in word duration to produce the same amount of change in reading accuracy. With respect to our analysis, we found that the slope of the psychometric function became

Table 5 | $\chi^{2}$ difference test of model $\boldsymbol{M}_{\text {fixed }}$ vs. model $\boldsymbol{M}_{\text {var }}$ for each participant. The nested model $\left(M_{\text {fixed }}\right)$ has two fewer degrees of freedom: linear and quadratic coefficients for the modulation of slope over the course of training.

\begin{tabular}{lrcc}
\hline Participant & $\Delta$ Deviance & $\boldsymbol{p}$-value & Significance \\
\hline S1 & 12.034 & 0.002 & $* *$ \\
S2 & 7.997 & 0.018 & $*$ \\
S3 & 14.002 & 0.001 & $* * *$ \\
S4 & 11.777 & 0.003 & $* *$ \\
S5 & 10.879 & 0.004 & $* *$ \\
S6 & 7.617 & 0.022 & $*$ \\
S7 & 2.226 & 0.329 &
\end{tabular}

measurably steeper with training for 5 out of 7 participants (see Figures 4, 5), although we acknowledge that there are individual variabilities.

The change in the slope of the psychometric function is interesting, and may be able to account for some improvements in reading performance. However, if the psychometric function simply becomes steeper but does not exhibit a shift toward shorter durations, then the function would be pivoted at the $50 \%$ point (as shown in Figure 1B), and we should observe a decrease in reading speed for reading accuracy below $50 \%$. Therefore, we also analyzed the data to determine if the improvements in reading performance occurred similarly across all durations, or only for some specific durations. As shown in Figure 7, the improvements in reading performance are not the same across all durations, ruling out scenario A in Figure $\mathbf{1}$ as the outcome of perceptual learning for most of the participants (S2-S6). The improvements are slightly larger near the high-end (performance close to $100 \%$ accuracy) of the psychometric function than the low-end (performance close to $0 \%$ accuracy). This finding, combined with the steepening of the psychometric function, identify scenario $\mathrm{C}$ as the effect of perceptual learning on the psychometric functions for our reading data.

In summary, following an RSVP training task to train participants with macular disease, we found that in addition to the previously reported improvement in reading speed, defined at the $80 \%$ accuracy, there is a steepening of the psychometric function relating reading accuracy with word exposure duration, accompanied by a shift of the psychometric function toward shorter duration. The shift is such that the psychometric function now appears to be pivoted at the low-end of the function. As such, the
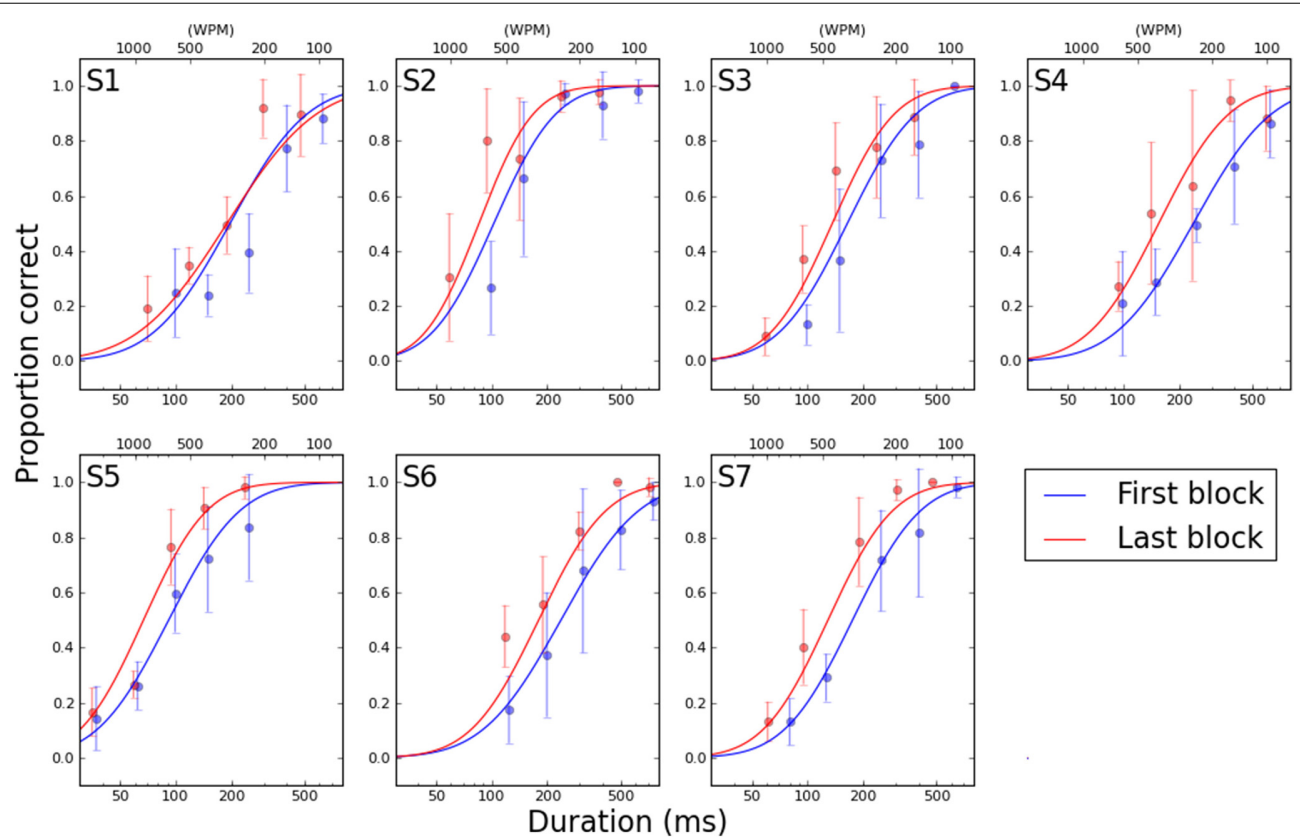

FIGURE 6 | First (blue curve) and last (red curve) psychometric functions for each participant, interpolated from model $\mathbf{M}_{\text {var }}$. Abscissa is word duration, shown in milliseconds on the bottom axis and equivalent reading speed in words per minute on the top axis. Points show the mean and standard deviation of the six repetitions of each word duration in the specified block. 

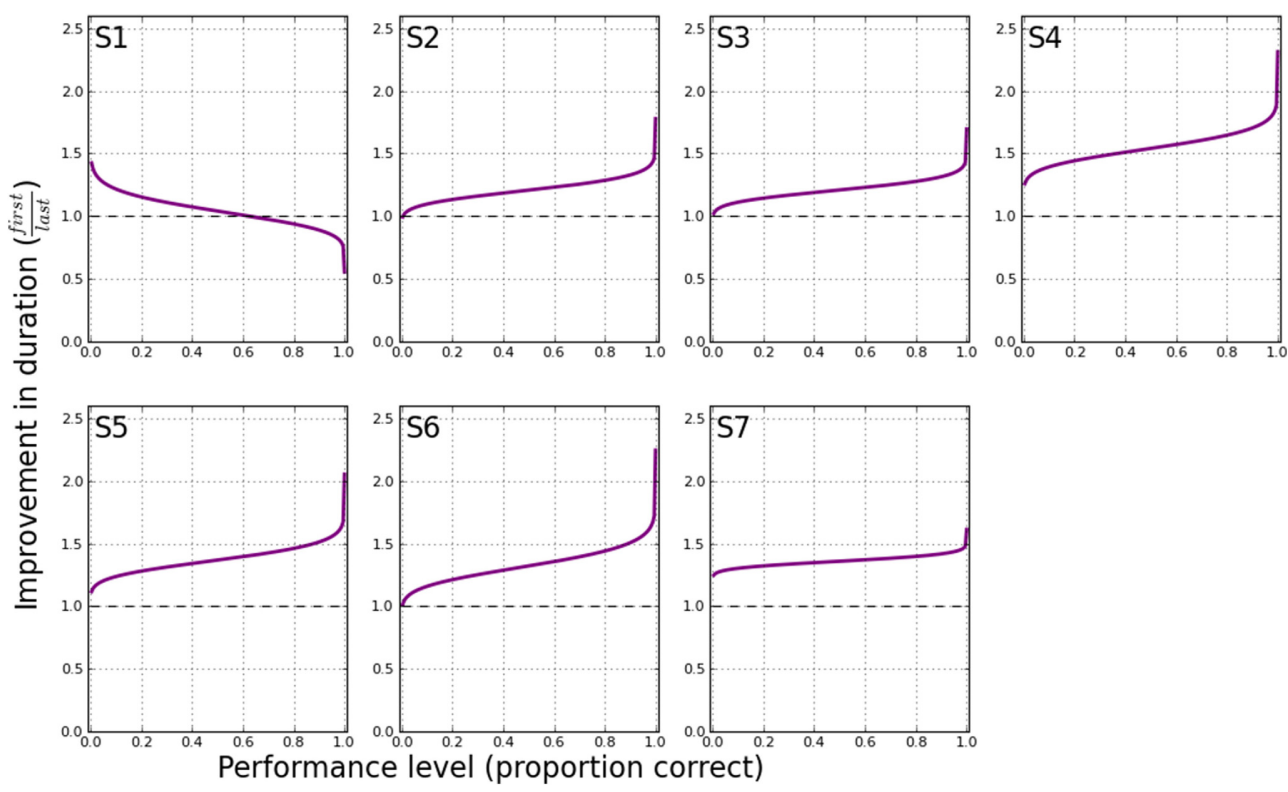

FIGURE 7 | Schematic improvement in performance (duration) at all points on the psychometric functions shown in Figure 6. At a given performance level (proportion correct), the corresponding stimulus durations are determined that yield the specified performance. The ratio of these durations (first/last) is then plotted on the ordinate, at the performance level indicated by the abscissa. magnitude of improvement in reading speed would depend on the criterion to define reading speed. For example, the improvement is generally slightly greater when reading speed is defined at $80 \%$ accuracy than at $50 \%$. This point is important for studies that use adaptive methods such as staircases for training, where reading performance is determined for more or less a similar accuracy level. Depending on the criterion accuracy level chosen, a larger or a smaller magnitude of improvement may be observed, and comparisons across studies would need to ensure that the accuracy levels are comparable.

\section{ACKNOWLEDGMENTS}

The authors would like to acknowledge significant influence from our reviewers, particularly for the statistical component of the manuscript. The authors were supported by NIH Research grant R01-EY012810.

\section{REFERENCES}

Astle, A. T., Webb, B. S., and McGraw, P. V. (2011). Can perceptual learning be used to treat amblyopia beyond the critical period of visual development? Ophthal. Physiol. Opt. 31, 564-573. doi: 10.1111/j.1475-1313.2011.00873.x

Ball, K., and Sekuler, R. (1982). A specific and enduring improvement in visual motion discrimination. Science 218, 697-698. doi: 10.1126/science.7134968

Ball, K., and Sekuler, R. (1987). Direction-specific improvement in motion discrimination. Vis. Res. 27, 953-965. doi: 10.1016/0042-6989(87)90011-3

Chung, S. T. L. (2002). The effect of letter spacing on reading speed in central and peripheral vision. Investig. Ophthalmol. Vis. Sci. 43, 1270-1276.

Chung, S. T. L. (2007). Learning to identify crowded letters: does it improve reading speed? Vis. Res. 47, 3150 - 3159. doi: 10.1016/j.visres.2007.08.017

Chung, S. T. L. (2011). Improving reading speed for people with central vision loss through perceptual learning. Invest. Ophthalmol. Vis. Sci. 52, 1164-1170. doi: 10.1167/iovs.10-6034

Chung, S. T. L., Legge, G. E., and Cheung, S.-H. (2004). Letter-recognition and reading speed in peripheral vision benefit from perceptual learning. Vis. Res. 44, 695-709. doi: 10.1016/j.visres.2003.09.028
Chung, S. T. L., Levi, D. M., and Tjan, B. S. (2005). Learning letter identification in peripheral vision. Vis. Res. 45, 1399-1412. doi: 10.1016/j.visres.2004. 11.021

Chung, S. T. L., Mansfield, J. S., and Legge, G. E. (1998). Psychophysics of reading. xviii. the effect of print size on reading speed in normal peripheral vision. Vis. Res. 38, 2949-2962. doi: 10.1016/S0042-6989(98)00072-8

Chung, S. T. L., and Truong, S. R. (2013). Learning to identify crowded letters: Does the learning depend on the frequency of training? Vis. Res. 77, 41-50. doi: 10.1016/j.visres.2012.11.009

Dosher, B. A., and Lu, Z. L. (2007). The functional form of performance improvements in perceptual learning: learning rates and transfer. Psychol. Sci. 18, 531-539. doi: 10.1111/j.1467-9280.2007.01934.x

Fahle, M., and Edelman, S. (1993). Long-term learning in vernier acuity: effects of stimulus orientation, range and of feedback. Vis. Res. 33, 397-412. doi: 10.1016/0042-6989(93)90094-D

Fahle, M., and Henke-Fahle, S. (1996). Interobserver variance in perceptual performance and learning. Investig. ophthalmol. Vis. Sci. 37, 869-877.

Karni, A., and Sagi, D. (1991). Where practice makes perfect in texture discrimination: evidence for primary visual cortex plasticity. Proc. Natl. Acad. Sci. 88, 4966-4970. doi: 10.1073/pnas.88.11.4966

Kilgarriff, A. (1997). Putting frequencies in the dictionary. Int. J. Lexicogr. 10, 135-155. doi: 10.1093/ijl/10.2.135

Knoblauch, K., and Maloney, L. T. (2012). Modeling Psychophysical Data in R. New York, NY: Springer.

Kontsevich, L. L., and Tyler, C. W. (1999). Bayesian adaptive estimation of psychometric slope and threshold. Vis. Res. 39, 2729-2737. doi: 10.1016/S00426989(98)00285-5

Levi, D. M. (1991). Spatial vision in amblyopia. Spat. Vis. 10, 212-238. doi: $10.1002 / \mathrm{dev} .20050$

Levi, D. M. (2005). Perceptual learning in adults with amblyopia: a reevaluation of critical periods in human vision. Dev. Psychobiol. 46, 222-232.

Levi, D. M., and Carkeet, A. D. (1993). "Amblyopia: a consequence of abnormal visual development," in Early Visual Development: Normal and Abnormal, ed K. Simons (New York, NY: Oxford University Press), 391-408.

Levi, D. M., and Li, R. W. (2009). Perceptual learning as a potential treatment for amblyopia: a mini-review. Vis. Res. 49, 2535-2549. doi: 10.1016/j.visres.2009.02.010 
Li, R. W., Levi, D. M., and Klein, S. A. (2004). Perceptual learning improves efficiency by re-tuning the decision'template'for position discrimination. Nat. Neurosc. 7, 178-183. doi: 10.1038/nn1183

Lu, Z.-L., and Dosher, B. A. (2004). Perceptual learning retunes the perceptual template in foveal orientation identification. J. Vis. 4:5. doi: 10.1167/4.1.5

McKee, S. P., and Westheimer, G. (1978). Improvement in vernier acuity with practice. Percept. Psychophys. 24, 258-262. doi: 10.3758/BF03206097

Millman, K. J., and Aivazis, M. (2011). Python for scientists and engineers. Compu. Sci. Eng. 13, 9-12. doi: 10.1109/MCSE.2011.36

Moscatelli, A., Mezzetti, M., and Lacquaniti, F. (2012). Modeling psychophysical data at the population-level: the generalized linear mixed model. J. Vis. 12:26. doi: $10.1167 / 12.11 .26$

Nguyen, N. X., Stockum, A., Hahn, G. A., and Trauzettel-Klosinski, S. (2011). Training to improve reading speed in patients with juvenile macular dystrophy: a randomized study comparing two training methods. Acta Ophthalmol. 89, e82-e88. doi: 10.1111/j.1755-3768.2010.02081.x

Owsley, C., McGwin, G., Lee, P. P., Wasserman, N., and Searcey, K. (2009). Characteristics of low-vision rehabilitation services in the united states. Arch. Ophthalmo. 127, 681-689. doi: 10.1001/archophthalmol.2009.55

Pérez, F., and Granger, B. E. (2007). IPython: a system for interactive scientific computing. Comput. Sci. Eng. 9, 21-29. doi: 10.1109/MCSE.2007.53

Poggio, T., Fahle, M., and Edelman, S. (1992). Fast perceptual learning in visual hyperacuity. Science 256, 1018-1021. doi: 10.1126/science. 1589770

R Core Team (2014). R: A Language and Environment for Statistical Computing. Vienna: R Foundation for Statistical Computing.

Rubin, G. S., and Turano, K. (1994). Low vision reading with sequential word presentation. Vis. Res. 34, 1723-1733. doi: 10.1016/0042-6989(94)90129-5

Seiple, W., Grant, P., and Szlyk, J. P. (2011). Reading rehabilitation of individuals with amd: relative effectiveness of training approaches. Invest. Ophthalmol. Vis. Sci. 52, 2938-2944. doi: 10.1167/iovs.10-6137
Tarita-Nistor, L., Brent, M. H., Steinbach, M. J., Markowitz, S. N., and Gonzalez, E. G. (2014). Reading training with threshold stimuli in people with central vision loss: a feasibility study. Optom. Vis. Sci. 91, 86-96. doi: 10.1097/OPX.0000000000000108

Watson, A. B., and Pelli, D. G. (1983). Quest: a bayesian adaptive psychometric method. Percept. Psychophys. 33, 113-120. doi: 10.3758/BF032 02828

White, J. M., and Bedell, H. E. (1990). The oculomotor reference in humans with bilateral macular disease. Invest. Ophthalmol. Vis. Sci. 31, 1149-1161.

Wood, S. N. (2006). Generalized Additive Models: An Introduction with R. Boca Raton, FL: Chapman and Hall; CRC Press.

Conflict of Interest Statement: The authors declare that the research was conducted in the absence of any commercial or financial relationships that could be construed as a potential conflict of interest.

Received: 20 August 2014; accepted: 24 November 2014; published online: 23 December 2014.

Citation: Coates DR and Chung STL (2014) Changes across the psychometric function following perceptual learning of an RSVP reading task. Front. Psychol. 5:1434. doi: 10.3389/fpsyg.2014.01434

This article was submitted to Perception Science, a section of the journal Frontiers in Psychology.

Copyright (C) 2014 Coates and Chung. This is an open-access article distributed under the terms of the Creative Commons Attribution License (CC BY). The use, distribution or reproduction in other forums is permitted, provided the original author(s) or licensor are credited and that the original publication in this journal is cited, in accordance with accepted academic practice. No use, distribution or reproduction is permitted which does not comply with these terms. 